\title{
SIMULTANEOUS ALLOCATION OF MULTIPLE DISTRIBUTED GENERATION AND CAPACITORS IN RADIAL NETWORK USING GENETIC-SALP SWARM ALGORITHM
}

Purpose. In recent years, the problem of allocation of distributed generation and capacitors banks has received special attention from many utilities and researchers. The present paper deals with single and simultaneous placement of dispersed generation and capacitors banks in radial distribution network with different load levels: light, medium and peak using genetic-salp swarm algorithm. The developed genetic-salp swarm algorithm (GA-SSA) hybrid optimization takes the system input variables of radial distribution network to find the optimal solutions to maximize the benefits of their installation with minimum cost to minimize the active and reactive power losses and improve the voltage profile. The validation of the proposed hybrid genetic-salp swarm algorithm was carried out on IEEE 34-bus test systems and real Algerian distributed network of Djanet (far south of Algeria) with 112-bus. The numerical results endorse the ability of the proposed algorithm to achieve a better results with higher accuracy compared to the result obtained by salp swarm algorithm, genetic algorithm, particle swarm optimization and the hybrid particle swarm optimization algorithms. References 27, tables 10, figures 12.

Key words: genetic algorithm, salp swarm algorithm, real power losses, distributed generation, capacitors.

Цель. В последние годы задача размещения распределенной генерации и батарей конденсаторов привлекает особое внимание многих организаций и исследователей. В данной работе рассмотрены отдельное и совместное размещение распределенной генерации и батарей конденсаторов в радиальной распределительной сети при различных уровнях нагрузки: слабом, среднем и пиковом с использованием алгоритма генетического роя сальпов (genetic-salp swarm algorithm). Разработанный алгоритм гибридной оптимизации генетического роя сальпов (GA-SSA) использует системные входные переменные радиальной распределительной сети для поиска оптимальных решений с целью максимизации преимуществ их установки с минимальными затратами для минимизации потерь активной и реактивной мощности и улучшения профиля напряжения. Тестирование предложсенного алгоритма гибридной оптимизации генетического роя сальпов было проведено на экспериментальных 34-шинных системах IЕEЕ и реальной 112-ииной алжсирской распределенной сети Джанета (крайний юг Алюсира). Численные результаты подтверждают способность предложенного алгоритма достигать лучиих результатов с больщей точностью по сравнению $с$ результатом, полученным методом роя сальпов, генетическим алгоритмом, оптимизацией роя частиц и алгоритмами гибридной оптимизации роя частиц. Библ. 27, табл. 10, рис. 12.

Ключевые слова: генетический алгоритм, алгоритм роя сальпов, реальные потери мощности, распределенная генерация, конденсаторы.

Introduction. Radial distribution network provides a link between high voltage transmission network and consumer services. The operation and planning studies of a distribution network require a steady state condition of the system for various load demands [1]. Hence, the need for flexibility of power systems is present. Energy savings and environmental impact ave given impetus to the development of distributed generation (DG), expected to play an increasing role in the power system of the future. Because of the penetration of DGs, the use of these sources in distribution networks is increasing throughout the world [2].

In power systems, loss and reliability are two significant points among several factors that can be considered, especially in distribution systems. Actually two of the most important DG profits are loss reduction and reliability improvement [3]. Installation and operation of DG units is one of the regulators politics in recent years to reduce system losses and improve system efficiency [4].

Capacitors have been widely used in distribution systems to achieve different objectives. The most important are: improving the voltage profile and reducing the power loss in the radial distribution system, determining the optimal locations and sizes of capacitors. [5]. DGs and capacitors used alone or simultaneously with optimum installation and proper sizing have an affecting on reducing network loss and improving voltage profile, because the non-optimal location and size of dispersed generation and capacitors can result in an increase in system losses and costs.
The problem of optimal dispersed generation location and sizing is divided into two sub problems, where the optimal location for DG placement is the one and how to select the most suitable size is the second. Many researches were provided in recent years about optimal placement and sizing of the capacitor and DGs using different methaheuristic methods. Researchers have developed many interesting methods and solutions. The differences are about the problem, which is formulated, methodology and assumptions being made. Some of the methods mentioned in [6]. Simultaneous placement of distributed generation (DG) and capacitor is considered in radial distribution network with different load levels in objective to reduce the active and reactive power loss, to reduce the energy loss and improvement of voltage profile. Again, several optimization algorithms have been proposed such as Fuzzy-DE and Fuzzy-MAPSO methods is proposed in [7], firefly algorithm (FA) [8-10], particle swarm algorithm optimization (PSO) [11], discrete particle swarm optimization (DPSO) [12], Grey Wolf Optimizer (GWO) [13], genetic algorithm (GA) [14], cuckoo search algorithm (CSA) [15], gravitational search algorithm (GSA) [16], salp swarm algorithm (SSA) [17], genetic moth swarm algorithm (GMSA) [18] and biogeography-based optimization (BBO) [19].

A new combined algorithm is proposed to evaluate the DG site and size in distribution network. The location of DGs and capacitors banks is obtained by GA and its 
size is optimized by SSA. First the initial population for equipment (DGs and capacitors) size an and location are produced by random, then the load flow was run. Using the given cost function and benefit's was implemented to optimize the size of DGs and capacitors which was calculated by SSA for the known location. In the next step, the new location of DG was calculated.

The results showed that the proposed combined GA-SSA method is better than the GA and SSA in terms of solution quality and number of iterations.

In this paper, DGs and fixed capacitors banks are installed for two scenarios: singly and simultaneously to minimize the required power transmission network, with different load levels (light, medium and peak). Cost of equipment and benefits are included in the objective function. This algorithm is applied to the network IEEE34 bus test and the Algerian distributed network in Djanet (City in Southern Algeria) with 112-bus.

Problem statement and formulation. The aim of paper is to maximize the installation benefit of DGs and capacitors and minimize active and reactive power losses for operational powers of the installed equipment to have the efficiency of the GA-SSA algorithm. The objective function is given by the following function:

$$
F=\max \sum_{i=1}^{N} \text { Benefit }(i)-\min \sum_{i=1}^{N} \operatorname{Cost}(i),
$$

where $N$ is the number of equipment's (DGS and capacitors banks) installed in the radial distribution network,

$$
\sum_{i=1}^{N_{D G}} \text { Benefit }(i)=B C+B E+B L+B R,
$$

where $B C$ - benefit of cost reduction of active power purchased; $B E$ - benefit of energy loss reduction; $B L-$ benefit of power loss reduction; $B R$ - reactive power loss reduction benefit; $N_{D G}$ - the number of distributed generation

$$
\sum_{i=1}^{N} \operatorname{Cost}(i)=\sum_{i=1}^{N_{D G}}\left(D G_{c}(i)+D G_{m}(i)\right)+\sum_{i=1}^{N_{C}}\left(\operatorname{cap}_{c}(i)\right),
$$

where $D G_{C}$ - distributed generation installation cost; $D G_{m}$ - maintenance cost of DGs; $c a p_{c}$ - capacitor installation cost; $N_{C}$ - the number of capacitors banks.

Mathematical formulation of the total objective function involves more than one objective function to be optimized simultaneously.

It considers capacitor banks installation cost, active power losses of transmission lines and the cost of distributed generation sources.

The power demand is evaluated as

$$
B C=\sum_{i=1}^{N_{D G}} B C_{B}(i)
$$

where $B C_{B}$ is the benefit of cost reduction of active power purchased after DG installation.

The benefit of energy loss reduction is

$$
B E=\sum_{i=1}^{N_{D G}} B E_{B}(i)+\sum_{i=1}^{N_{C}} B E_{A}(i)
$$

where $B E_{B}$ and $B E_{A}$ are the benefit of energy loss reduction after DG installation and capacitor installation, respectively.
The peak power loss reduction is one of the most important effect of capacitor placements in distribution network and can be computed as

$$
B L=C_{d} \cdot\left(\sum_{i=1}^{N_{C}} B L_{B}(i)-\sum_{i=1}^{N_{C}} B L_{A}(i)\right),
$$

where $B L_{B}$ is the peak power loss in feeder before capacitor installation ( $\mathrm{kW}) ; B L_{A}$ is the peak power loss in feeder after capacitor installation $(\mathrm{kW}) ; C_{d}$ is demand reduction benefit rate $(\$ / \mathrm{MW})$.

The reduction of reactive power loss benefit can be calculated as

$$
B R=C_{R}\left(Q_{0}-Q_{1}\right),
$$

where $Q_{0}, Q_{1}$, and $C_{R}$, are reactive power loss before installation of equipment ( $\mathrm{kVar}$ ), reactive power loss after installation of equipment ( $\mathrm{kVar})$, and worth of reactive power $(\$ / \mathrm{kVar})$ respectively.

The cost of capacitor installation is given by:

$$
\operatorname{cap}_{c}=\operatorname{Cost}_{C i} \cdot n_{C i},
$$

where $\operatorname{Cost}_{C i}$ is the cost of $i$-th capacitor, $n_{C i}$ is the number of $i$-th capacitor.

The DG installation cost of distributed generation can be formulated as following equation:

$$
D G_{c}=\sum_{i=1}^{N_{D G}} D G_{c, i} \cdot K_{D G},
$$

where $K_{D G}$ is the size of DGs; $D G_{c, i}$ is the installation cost of $i$-th DGs.

The maintenance cost of distributed generation is formulated as

$$
D G_{m}=\sum_{i=1}^{N_{D G}} D G_{m, i} \cdot K_{D G},
$$

where $D G_{m, i}$ is the maintenance cost of $i$-th DGs.

Optimal location and sizing of DGs and capacitors. The optimal location and sizing problems of distributed generation and capacitors bank are bi-objective constrained optimization problems that can be formulated as a monoobjective constrained optimization problem. In this paper the algorithm genetic-salp swarm has been developed for solving the problems of optimal location and sizing of distributed generation and capacitors banks in radial distribution network by maximizing the benefits and minimizing the costs of installation for reduce the active and reactive power losses and improve the voltage profile.

Basic concepts of genetic algorithm. Genetic algorithms are based on chromosomes and their natural evolution. In this method, contrary to the analytical methods, try and error, instead of working on an optimal answer, they work on several answers that they call «populations». These methods is based on the selection mechanisms used by nature, according to which the most fit individuals of a population are those that survive, by adapting more easily to the changes that occur in their environment. This technique has high efficiency in the absence of specific information about the problem [21, 22].

The parameters of genetic algorithm are: population size, reproduction probability, crossover probability and mutation probability. follows: 
Step 1: Initialize population in random fashion.

Step 2: Evaluate the population by calculating the fitness of the individuals in the population.

Step 3: Perform selection operation.

Step 4: Perform crossover and mutation operation.

Step 5: Perform optimal search.

Step 6: Repeat step $2-5$ until the GA is run for the predetermined.

Step 7: Select the best chromosome.

Basic concepts of salp swarm algorithm. Salp swarm algorithm (SSA) is a recently created bio-inspired optimization algorithm presented in 2017, introduced by Mirjalili et al. [23]. SSA mimics the swarming behavior of salps in oceans especially the navigation and searching for food sources. The salps are creatures living in seas and oceans. They are similar to jellyfishes in their tissues and movement towards the food sources [24]. Salps are usually found in groups (swarms) called salp chains; each salp chain contains a leader .The algorithm starts with initializing a matrix with a dimension of $n \cdot d i m$ representing salps' positions, where $n$ is the number of agents and $\operatorname{dim}$ is the number of variables to be designed. After that, each salp position is updated according to instructions received from the leader for swallowing the best food $(F)$. The following equation is used for updating the salps' positions [25]:

$$
x_{j}^{1}=\left\{\begin{array}{l}
F_{j}+c_{1}+\left(\left(u_{b_{j}}-l_{b_{j}}\right) c_{2}+l_{b_{j}}\right), c_{2} \geq 0 ; \\
F_{j}-c_{1}+\left(\left(u_{b_{j}}-l_{b_{j}}\right) c_{2}+l_{b_{j}}\right), c_{2} \leq 0,
\end{array}\right.
$$

where $x_{j}^{1}$ represents the position of the leader's $j$-th dimension; $F_{j}$ represents the best solution (i.e., food source) in the $j$-th, $u_{b_{j}}$ and $l_{b_{j}}$ are the upper and lower bounds in the $j$-th dimension respectively; variables $c_{1}$ and $c_{2}$ are random numbers $\left(c_{1}\right.$ plays a vital role in the performance of SSA, since it is the only parameter that controls the balance between exploration and exploitation).

As can be seen in [12], $c_{1}$ is a time varying parameter (depends on the iteration number) that allows high exploration rates at the early stages of the optimization process, while high exploitation rates are allowed in the last stages. The coefficient is a very significant parameter in SSA for the reason that it maintains a balance between exploration and exploitation, and it can be given by:

$$
c_{1}=2 e^{-\left(\frac{4 l}{L}\right)^{2}}
$$

where $l$ and $L$ are the current iteration and the maximum number of iterations respectively; $c_{2}$ is uniform random numbers generated in the period $[0,1]$.

These variables indicate whether the next position in the $j$-th dimension will be moved towards the $+\infty$ or $-\infty$ in addition to the step size. Newton's law of motion [14], was used to simulate updating the positions of the followers

$$
x_{j}^{1}=\frac{1}{2}\left(x_{j}^{i}+x_{j}^{i-1}\right),
$$

where $i \geq 2$ and $x_{j}^{i}$ depicts the position of the $i$-th follower at the $j$-th dimension.

In SSA, the leader salp moves towards the food source, whereas the followers move towards the leader.
The food source position can be changed during the process and then the leader will continue moving towards the new food source position. The steps of this optimization algorithm and its pseudo code are shown in Algorithm 1 below.

Generate an initial population of salps randomly

$x_{i}(i=1,2, \ldots, n)$ considering upper bound $u_{b}$ and lower

bound $l_{b}$

While $(t<$ maximum number of iteration) do

Calculate the fitness function of each salp $\left(f\left(x_{i}\right)\right.$

Set the best salp to $x_{\text {best }}$

Update the value of $c_{1}$ according to Eq. [13]

For (each salp $\left.\left(x_{i}\right)\right)$ do

If $x_{i}$ is the leader then

Update the position of leading salp using Eq. [12]

Else

Update the position of the follower salp using Eq. [14]

End if

Update the position of the salp

End for

Update the position of the salps based on the upper and lower bounds

End while

Return the best salp $x_{\text {best }}$

Improvement method GA-SSA. This is a searching technique developed for optimal location and sizing of DG and capacitors. The problem consists of 2 parts. The first is the optimal location of DG and capacitors and the second is the optimal location. Result for the first part is an integer, which is either a bus number where DGs and capacitors are suggested to be installed. This needs an integer-based optimization algorithm. GA has been chosen to play this role because of its attractive quality. The answer obtained from GA solution is used in SSA algorithm to optimize the sizing for DG. SSA has the fast convergence ability, which is a great attractive property for a large iterative and time consuming problem. Therefore the algorithm is recovered in 16 steps:

Step 1: Read data from network (,$X$, PLoad, QLoad).

Step 2: Determine the number of chromosomes, generation, and mutation rate and crossover rate value.

Step 3: Initial set of random size of DG and capacitors.

Step 4: Initial set of random location of DG and capacitors.

Step 5: Calculate the objective values (Parts of objective function in different load levels).

Step 6: Calculate the loss of active and reactive power losses in different load levels .

Step 7: Using SSA to calculate fitness for optimal sizing of DG and capacitors.

Step 8: Evaluation of fitness value of chromosomes by calculating objective function.

Step 9: Using GA to calculate fitness for optimal sitting of DG (Selection - Crossover - Mutation).

Step 10: Calculate the objective values (Parts of objective function in different load levels).

Step 11: Calculate the loss of active and reactive power losses in different load levels.

Step 12: Using SSA to calculate fitness for optimal sizing of DG and capacitors. 
Step 13: Searching for best solution.

Step 14: Check the stop Criterion.

Step 15: If the stopping criteria is satisfied then stop, else go to step 5 .

Step 16: Best solution.

Simulations and results. The hybrid GA-SSA technique for optimal location and sizing of DGs and capacitors have been implemented in MATLAB 7.10 programming language and the simulation conducted on a computer Core (TM) i5 a $1.90 \mathrm{GHz}$ with 8 Go RAM. This algorithm tested in IEEE 34-bus [20] radial distribution system and the real network 112-bus radial distribution system [26] shown in Fig. 1 and Fig. 2 respectively.

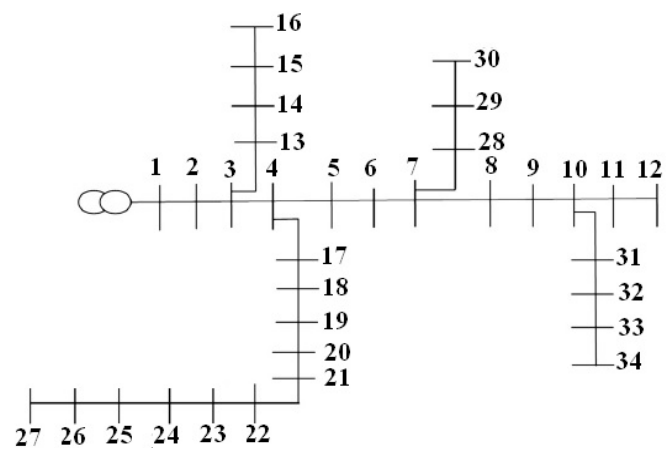

Fig. 1. IEEE 34-Bus test system

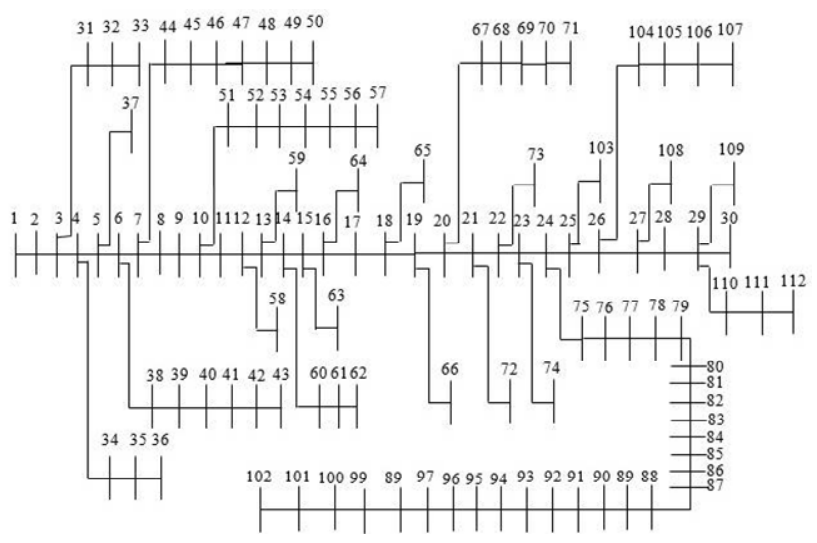

Fig. 2. Real network 112-bus

IEEE 34-Bus radial distribution network. The active and reactive power demand in peak load are $11303 \mathrm{KW}$ and 7044.2 KVar respectively. The capacitors are installed in the system such that the reactive power generated by the maximum total capacity does not exceed $20 \%$ of the reactive power in peak load [27]. That corresponds to reactive power 1408.84 KVar. We therefore chose to install 7 capacitors with $200 \mathrm{KVar}$ each one at optimal locations in order to maximize the objective function equation [1].

Three different load levels are considered in this paper: light, medium and peak load. The load growth rate is considered $1 \%$ in each year. Tables 1 shows technical and commercial information of 3 load duration (h/year) and electricity market price $(\$ / \mathrm{MWh})$ and Table 2 shows the parts of the objective function.

Now, in order to minimize active and reactive power losses and improve the voltage profile, 4 cases are considered. Figure 3 shows the locations and sizes of the capacitor banks as well as the distributed generators used for the 4 cases which are as follows:
- Case 1: without installing equipment's.

- Case 2: only 7 capacitors of $200 \mathrm{KVar}$ are optimally placed at bus 3, 7, 10, 15, 24, 31 and 34 .

- Case 3: only 5 DGs are installed. 4 DGs are optimally placed at bus 4, 8, 22 and 24 with capacity of $500 \mathrm{KW}$, and one DG at bus 33 with capacity of $250 \mathrm{KW}$.

- Case 4: 5 DGs and 7 capacitors are installed simultaneously with same optimal placement.

Table 1

Technical and commercial information of load and electricity market price

\begin{tabular}{|c|c|c|}
\hline Network condition & $\begin{array}{c}\text { Time duration } \\
(\mathrm{h} / \text { year })\end{array}$ & $\begin{array}{c}\text { Market price } \\
(\$ / \mathrm{MWh})\end{array}$ \\
\hline Light load & 2190 & 35 \\
\hline Medium load & 4745 & 49 \\
\hline Peak load & 1825 & 70 \\
\hline
\end{tabular}

Table 2

Parts of objective function in different load levels

\begin{tabular}{|l|c|c|}
\hline \multicolumn{1}{|c|}{ Economical cost } & Network condition & Costs (\$) \\
\hline Capacitor installation cost & \multirow{3}{*}{ Light load } & 14000 \\
DG installation cost & 397500 \\
DG maintenance cost & 15330 \\
\hline Capacitor installation cost & \multirow{3}{*}{ Medium load } & 1426830 \\
\hline DG installation cost & & 397500 \\
\hline DG maintenance cost & & 33215 \\
\hline Capacitor installation cost & \multirow{2}{*}{ Peak load } & 144715 \\
\hline DG installation cost & 397500 \\
\hline DG maintenance cost & 12775 \\
\hline \multicolumn{2}{|c|}{} & 424275 \\
\hline
\end{tabular}

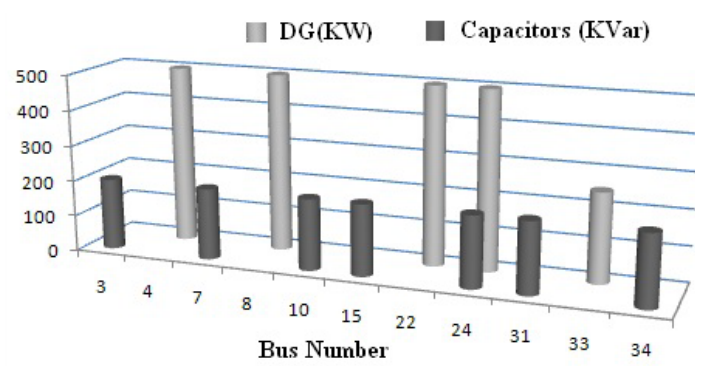

Fig. 3. Location and size of DGs and capacitors installed in IEEE 34-Bus

The optimum sizes of DGs placed respectively at optimal bus, obtained to minimize active and reactive power losses and improve the voltage profile are:

- in case 3 DGs values are determined as $412.19 \mathrm{KW}$, 86.53 KW, 431.82 KW, 463.02 and $88.14 \mathrm{KW}$ but the optimal size of all DGs in case 4 are $405.06 \mathrm{KW}$, 151.21 KW, 252.43 KW, 355.50 and $76.27 \mathrm{KW}$ in bus 4, $8,22,24$ and 33 respectively.

These results are presented in Fig. 4.

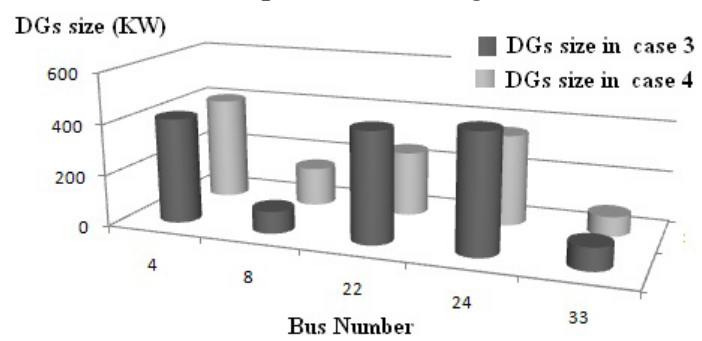

Fig. 4. Location and size of DGs in case 3 and 4 
Table 3 represents the loss of active and reactive power in the 4 cases considered with the 3 load levels: light, medium and peak load.

Table 3

The loss of active and reactive power in the four cases considered with the three load levels

\begin{tabular}{|c|c|c|c|}
\hline \multirow{4}{*}{$\begin{array}{c}\text { Without } \\
\text { equipment's }\end{array}$} & $\begin{array}{c}\text { Load } \\
\text { level }\end{array}$ & $\begin{array}{c}\text { Active power } \\
\text { losses (kW) }\end{array}$ & $\begin{array}{c}\text { Reactive power } \\
\text { losses (KVar) }\end{array}$ \\
\cline { 2 - 4 } & $\begin{array}{c}\text { Might load } \\
\text { load }\end{array}$ & 512.6674 & 140.9201 \\
\cline { 2 - 4 } & Peak load & 1136.6012 & 311.7137 \\
\hline \multirow{3}{*}{$\begin{array}{c}\text { With only } \\
\text { capacitors }\end{array}$} & \begin{tabular}{c} 
Might load \\
\cline { 2 - 4 } Wedium \\
load
\end{tabular} & 498.2234 & 116.5629 \\
\cline { 2 - 4 } & Peak load & 1005.4012 & 282.4204 \\
\hline \multirow{3}{*}{$\begin{array}{c}\text { With DGs and } \\
\text { capacitors }\end{array}$} & Light load & 267.3280 & 59.5580 \\
\cline { 2 - 4 } & $\begin{array}{c}\text { Medium } \\
\text { load }\end{array}$ & 270.2497 & 61.4683 \\
\cline { 2 - 4 } & Peak load & 676.1747 & 189.2695 \\
\cline { 2 - 4 } & Might load & 178.9525 & 42.7015 \\
\cline { 2 - 4 } & Pead & 205.4583 & 50.9711 \\
\hline
\end{tabular}

Note that for Case 2, the loss of active and reactive power are reduced by $2.81 \%, 11.63 \%, 11.54 \%$ (Table 4) and $17.28 \%, 25.17 \%, 9.39 \%$ (Table 5), respectively, and for 3 load levels respectively as compared with the case 1 .

Case 3, the loss of active and reactive power are more reduced than the case 2 of $47.85 \%, 57.92 \%$, $40.50 \%$ (Table 4) and $57.73 \%, 65.16 \%, 39.28 \%$ (Table 5) respectively, and three load levels respectively as compared to the case 1 .

Case 4 , the loss of active and reactive power are even more reduced than cases 2 and 3, 65.09\%, 68.01\%, $68.42 \%$ (Table 4) and $69.70 \%, 71.11 \%, 69.09 \%$ (Table 5), respectively and for 3 load levels respectively as compared to the case 1 .

Table 4

Benefits of active power losses reduction

\begin{tabular}{|c|c|c|c|}
\hline & Light load & Medium load & Peak load \\
\hline Case 2 & $2.81 \%$ & $11.63 \%$ & $11.54 \%$ \\
\hline Case 3 & $47.85 \%$ & $57.92 \%$ & $40.50 \%$ \\
\hline Case 4 & $65.09 \%$ & $68.01 \%$ & $68.42 \%$ \\
\hline
\end{tabular}

Table 5

Benefits of reactive power losses reduction

\begin{tabular}{|c|c|c|c|}
\hline & Light load & Medium load & Peak load \\
\hline Case 2 & $17.28 \%$ & $25.17 \%$ & $9.39 \%$ \\
\hline Case 3 & $57.73 \%$ & $65.16 \%$ & $39.28 \%$ \\
\hline Case 4 & $69.70 \%$ & $71.11 \%$ & $69.09 \%$ \\
\hline
\end{tabular}

Table 6 represents a comparative study between the results obtained by the proposed algorithm GA-SSA and those obtained by SSA, GA, PSO (particle swarm optimization) and the HPSO (hybrid particle swarm optimization) algorithms [27].

Table 6

Comparative study in medium load

\begin{tabular}{|c|c|c|c|c|c|}
\hline Size & GA-SSA & GA & SSA & PSO & HPSO \\
\hline $\begin{array}{c}\text { DG size } \\
\text { in total (kW) }\end{array}$ & 1240.47 & 1423.78 & 1283.14 & 1653.62 & 1493.56 \\
\hline $\begin{array}{c}\text { Capacitor size } \\
\text { in total (KVar) }\end{array}$ & 1400 & 2126 & 1400 & 2542 & 2452 \\
\hline $\begin{array}{c}\text { Power } \\
\text { losses (KW) }\end{array}$ & 527.55 & 608.84 & 612.55 & 620.64 & 614.55 \\
\hline
\end{tabular}

We note that the loss of active power obtained by the GA-SSA algorithm is $8.55 \%$ better than those values obtained by PSO algorithm and $7.65 \%$ than those obtained by algorithm HPSO. Thus of viewpoint of production cost, reactive power obtained by GA-SSA (1400 kVA) is also better than that obtained by PSO (2542 kVA) and HPSO (2452 kVA).

This comparison clearly demonstrates the effectiveness of the proposed genetic salp swarm algorithm compared to genetic algorithm, salp swarm algorithm, particle swarm optimization and hybrid particle swarm optimization algorithm.

Fig. 5-8 represents the voltage profile at the distribution network for the 4 cases considered and for the 3 load levels. Table 6 shows the minimum voltage in the distribution network for the 4 cases considered and the 3 load levels. The values obtained for peak load: $0.8857 \mathrm{pu}$ at bus 27 case $1,0.9390$ pu at bus 27 case 2, 0.9403 pu at bus 24 case 3 and 0.9647 pu at bus 27 case 4 show good improvement under voltage where the values of the voltage obtained are between 0.9647 in the different load levels: light, medium and peak.

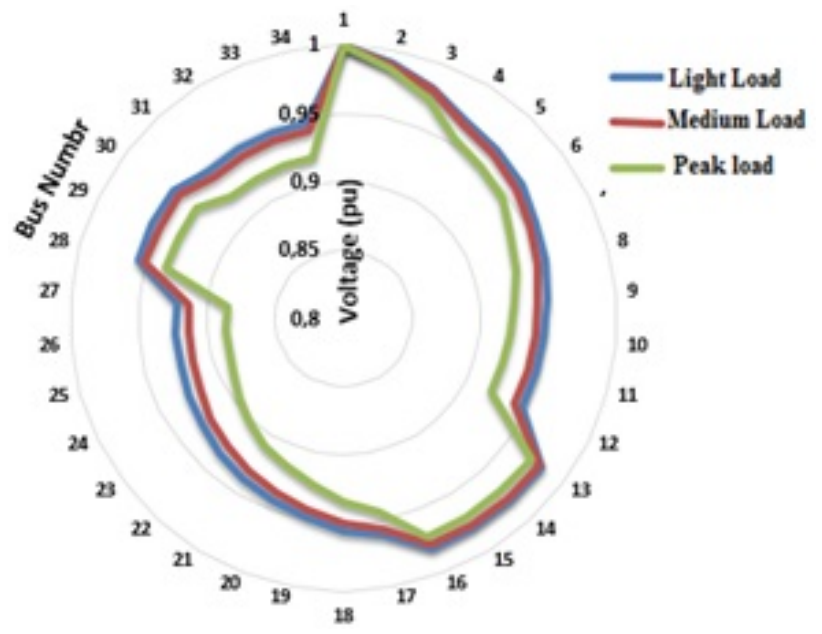

Fig. 5. Performance of voltage profile for 34 bus radial distribution in different load levels without installation of capacitors and DGs

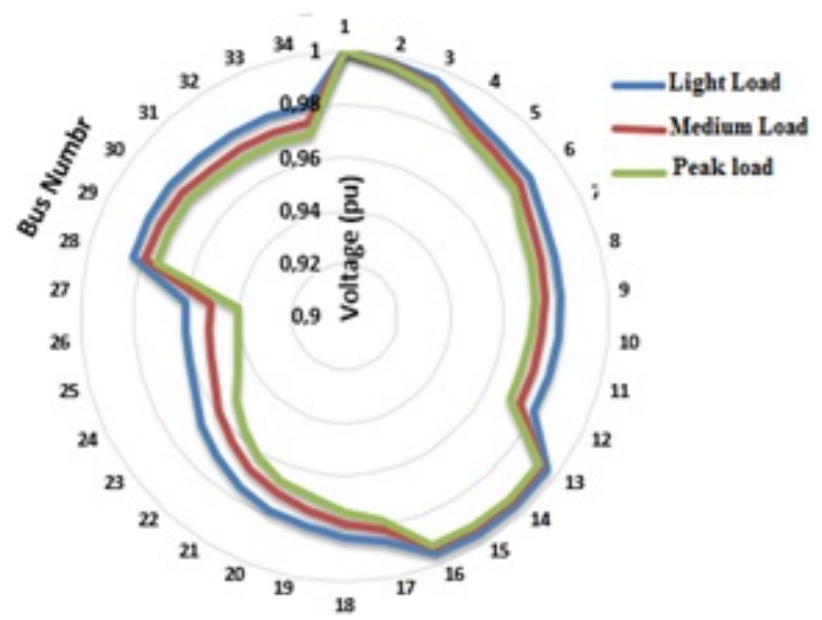

Fig. 6. Performance of voltage profile for 34 bus radial distribution in different load levels after installation of capacitors 


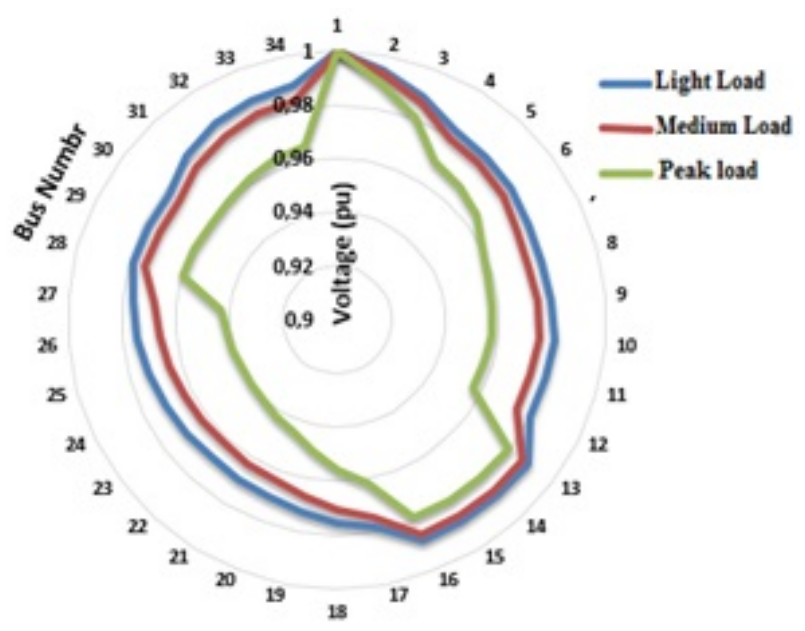

Fig. 7. Performance of voltage profile for 34 bus radial distribution in different load levels after installation of DGs

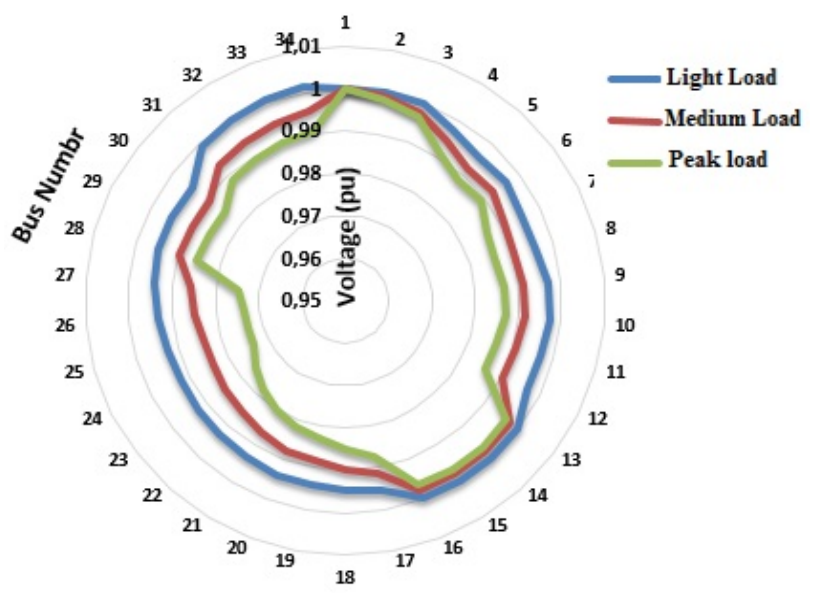

Fig. 8. Performance of voltage profile for 34 bus radial distribution in different load levels after installation of capacitors and DGs

Real distribution network of 112-Bus. The active and reactive power demand in peak load are $11303 \mathrm{KW}$ and 7044.2 KVar respectively. Table 7 shows the parts of objective function in different load levels.

Parts of objective function in different load levels

Table 7 of real network 112-bus

\begin{tabular}{|c|c|c|}
\hline Economical cost & Network condition & Costs $(\$)$ \\
\hline Capacitor installation cost & \multirow{3}{*}{ Light load } & 14400 \\
\hline DG installation cost & & 357120 \\
\hline DG maintenance cost & & 18396 \\
\hline Total & & 389916 \\
\hline Capacitor installation cost & \multirow{3}{*}{ Medium load } & 14400 \\
\hline DG installation cost & & 477000 \\
\hline DG maintenance cost & & 39858 \\
\hline Total & & 531258 \\
\hline Capacitor installation cost & \multirow{3}{*}{ Peak load } & 14400 \\
\hline DG installation cost & & 477000 \\
\hline DG maintenance cost & & 15330 \\
\hline Total & & 506730 \\
\hline
\end{tabular}

The 4 cases studied to have the effectiveness of the method in the real 112-bus network (Fig. 2) are:

- Case 1: without installing equipment's.

- Case 2: in this case, we have installed 6 capacitors with $150 \mathrm{KVar}$. The optimal locations are obtained at the bus: 4, 25, 34, 38, 79 and 98 .

- Case 3: we have installed 6 DGs: 2 DGs are optimally placed at bus 4 and 33 with capacity of $500 \mathrm{KW}$, and $4 \mathrm{DG}$ at bus 33, 58, 88 and 92 with capacity of $250 \mathrm{KW}$.

- Case 4: 5 capacitors are installed with optimal placement at bus $6,25,34,56$ and 102 with $150 \mathrm{KV}$ ar and 4 DGs with optimal locations obtained at the bus: 12, 45, 76 and 92 with capacity of $250 \mathrm{KW}$.

The comparison between the results obtained by the GA-SSA algorithm and the SSA and GA algorithms are represented in Table 8.

Table 8

Comparison between the results obtained by the GA-SSA

algorithm and other algorithms (real network 112-bus)

\begin{tabular}{|c|c|c|c|c|c|}
\hline \multicolumn{2}{|c|}{ Case } & $\begin{array}{c}V_{\min } \\
(\mathrm{p} . \mathrm{u})\end{array}$ & $\begin{array}{c}P_{\text {loss }} \\
(\mathrm{KW})\end{array}$ & $\begin{array}{c}Q_{\text {loss }} \\
(\mathrm{KV} \text { ar })\end{array}$ & $\begin{array}{c}\text { CPU } \\
\text { time (s) }\end{array}$ \\
\hline \multicolumn{2}{|c|}{ Base Case } & 0.9314 & 288.14 & 189.64 & - \\
\hline \multirow{3}{*}{$\begin{array}{c}\text { With only } \\
\text { capacitors }\end{array}$} & GA-SSA & 0.9394 & 224.16 & 174.22 & 36 \\
\cline { 2 - 6 } & GA & 0.9377 & 236.09 & 182.52 & 66 \\
\cline { 2 - 6 } & SSA & 0.9381 & 228.12 & 179.01 & 42 \\
\hline \multirow{4}{*}{\begin{tabular}{l} 
With only DGs \\
\cline { 2 - 6 }
\end{tabular}} & GA & 0.9569 & 124.96 & 102.19 & 37 \\
\cline { 2 - 6 } & SSA & 0.9498 & 136.08 & 142.71 & 74 \\
\hline \multirow{3}{*}{$\begin{array}{c}\text { With capacitors } \\
\text { and DGs }\end{array}$} & GA-SSA & 0.9802 & 56.32 & 137.24 & 48 \\
\cline { 2 - 6 } & GA & 0.9684 & 96.37 & 71.27 & 39 \\
\cline { 2 - 6 } & SSA & 0.9776 & 82.06 & 68.62 & 52 \\
\hline
\end{tabular}

The benefits of active and reactive power losses reduction of cases $2-4$ are compared with those of case 1 for 112-bus are presented in Table 9, 10, respectively.

Table 9

Benefits of active power losses reduction compared with the case 1 in real network 112-bus

\begin{tabular}{|c|c|c|c|}
\hline & \multicolumn{3}{|c|}{ Benefits of active power losses reduction } \\
\hline Load level & Light load & Medium load & Peak load \\
\hline Case 2 & $38.81 \%$ & $22.20 \%$ & $30.64 \%$ \\
\hline Case 3 & $64.85 \%$ & $56.63 \%$ & $68.50 \%$ \\
\hline Case 4 & $96.09 \%$ & $80.45 \%$ & $74.42 \%$ \\
\hline
\end{tabular}

Table 10

Benefits of reactive power losses reduction compared with the case 1 in real network 112-bus

\begin{tabular}{|c|c|c|c|}
\hline & \multicolumn{3}{|c|}{ Benefits of reactive power losses reduction } \\
\hline Load level & Light load & Medium load & Peak load \\
\hline Case 2 & $17.28 \%$ & $8.13 \%$ & $9.39 \%$ \\
\hline Case 3 & $57.73 \%$ & $46.11 \%$ & $39.28 \%$ \\
\hline Case 4 & $79.70 \%$ & $77.36 \%$ & $69.09 \%$ \\
\hline
\end{tabular}

Fig. 9 represents the voltage profile of the real network 112-bus without installation of the equipment where the minimum values of the voltage for the 3 load levels, peak, medium and light are respectively: 0.9114, $0.9316,0.9303$.

Fig. 10, corresponding to case 2, represents the real 112-bus network voltage profile after the installation of capacitors where the minimum voltage values for the 3 load levels, peak, medium and light are respectively $0.9343,0.9394$ and 0.9422 . 
The installation of the capacities also allowed a reduction of active power losses of $38.82 \%, 22.20 \%$, $30.64 \%$ and a reduction of reactive power losses of $17.28 \%, 8.13 \%, 9.39 \%$ compared to case 1 , respectively for the 3 load levels.

Fig. 11, corresponding to case 3, represents the real network 112-bus network voltage profile after installation of DGs where the minimum voltage values for the 3 load levels, peak, medium and light are respectively 0.9535 , 0.9569 and 0.9578 .

The installation of the DGs also allows a reduction of active power losses of $64.85 \%, 56.36 \%, 68.50 \%$ and a reduction of reactive power losses of $57.73 \%, 46.11 \%$, $39.28 \%$ compared to case 1 respectively for the 3 load levels.

Fig. 12, corresponding to case 4 , represents the real network 112-bus network voltage profile after simultaneous installation of DGs and capacitors where the minimum voltage values for the 3 load levels, peak, medium and light are 0.9792, 0.9802 and 0.9812 respectively.

The installation of the 2 equipment allowed a further reduction of active power losses of $96.09 \%, 80.45 \%$ and $74.42 \%$ and a reduction of reactive power losses of $79.70 \%, 77.36 \%$ and $69.09 \%$ compared to case 1 respectively for the 3 load levels.

The results obtained in this scenario are better than those obtained in case 2 and case 3 from the point of view of the voltage profile and the maximization of the benefit of the DGs installation and capabilities.

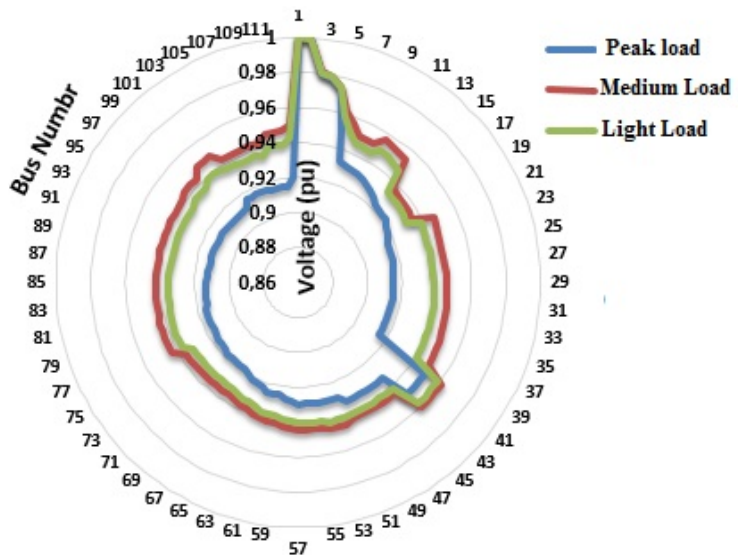

Fig. 9. Performance of voltage profile for real network 112-bus in different load levels without installation of capacitors and DGs

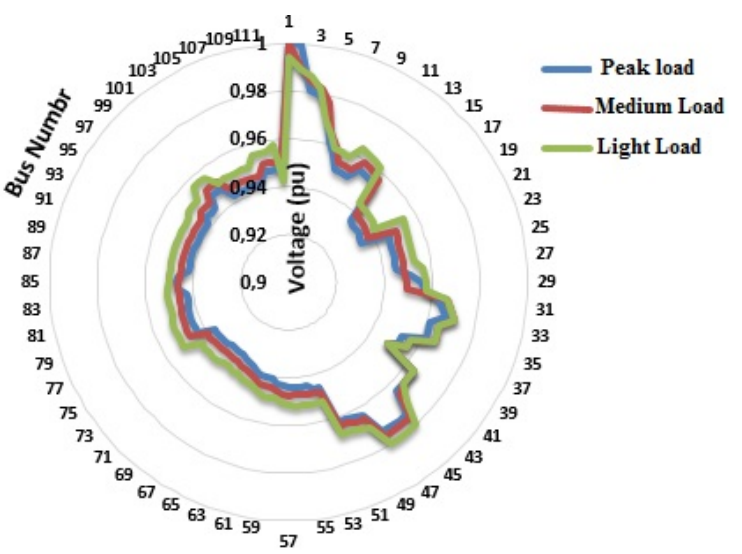

Fig. 10. Performance of voltage profile for real network 112-bus in different load levels after installation of capacitors

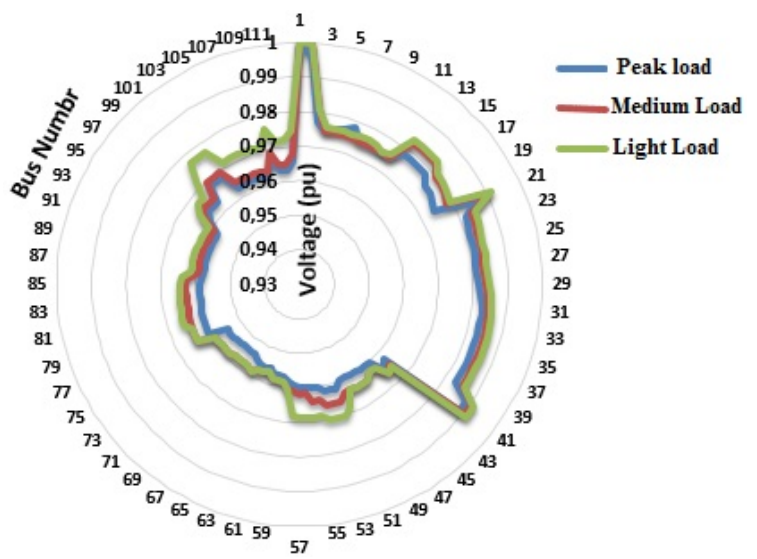

Fig. 11. Performance of voltage profile for real network 112-bus in different load levels after installation of DGs

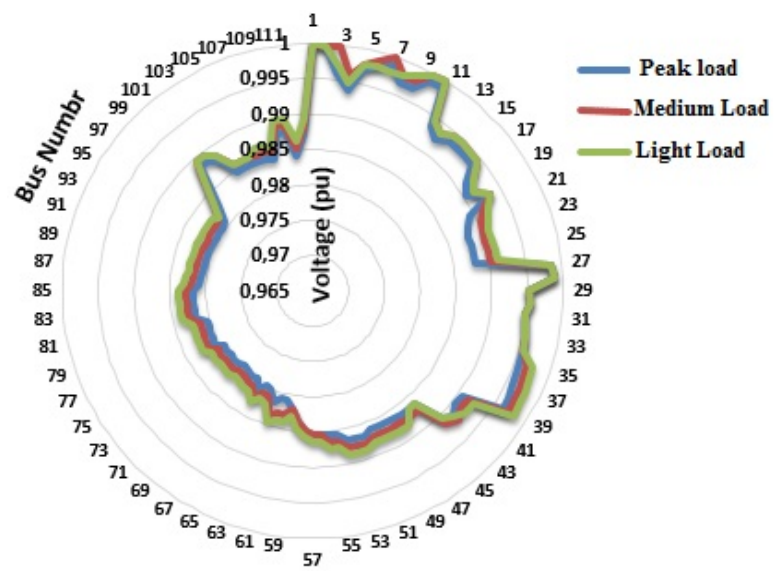

Fig. 12. Performance of voltage profile for real network 112-bus in different load levels after installation of capacitors and DGs

Conclusion. In this article, two algorithms genetic and salp swarm algorithm have been proposed for the simultaneously optimal placement of distributed generation and capacitors in a distribution system. These two algorithms are applied on four different cases. The purpose of this combination is to maximize the installation benefit of distributed generation and capacitor banks and minimize active and reactive power losses in radial distribution network, also to minimize the computation time, and to seek the optimal overall solution. This method is applied on radial distribution network IEEE 34-bus and real Algerian distributed network of Djanet 112-bus, the result shows that, cases with simultaneous placement of distributed generation and capacitor have much improvement of voltage profile and power loss reduction compared with others methods.

\section{REFERENCES}

1. Satyakar V.V.K., Viswanatha Rao J., Manikandan S. Analysis of radial distribution system by optimal placement of DG using DPSO. International Journal of Engineering Research \& Technology, 2012, vol. 1, iss. 10, pp. 1-7.

2. Khanjanzadeh A., Arabi M., Sedighizadeh M., Rezazadeh A. Distributed generation allocation to improve steady state voltage stability of distribution networks using particle swarm optimization and genetic algorithm. Canadian Journal on Electrical and Electronics Engineering, 2011, vol. 2, no. 6.

3. Herbadji O., Nadhir K., Slimani L., Bouktir T. Optimal power flow with emission controlled using firefly algorithm. 
2013 5th International Conference on Modeling, Simulation and Applied Optimization (ICMSAO), Apr. 2013. doi: 10.1109/icmsao.2013.6552559.

4. Gkaidatzis P.A., Bouhouras A.S., Doukas D.I., Sgouras K.I., Labridis D.P. Load variations impact on optimal DG placement problem concerning energy loss reduction. Electric Power Systems Research, 2017, vol. 152, pp. 36-47. doi: 10.1016/j.epsr.2017.06.016.

5. Baghipour R., Hosseini S.M. Placement of DG and capacitor for loss reduction, reliability and voltage improvement in distribution networks using BPSO. International Journal of Intelligent Systems and Applications, 2012, vol. 4, no. 12, pp. 57-64. doi: 10.5815/ijisa.2012.12.08.

6. Kumar Injeti S., Shareef S.M., Kumar T.V. Optimal allocation of DGs and capacitor banks in radial distribution systems. Distributed Generation \& Alternative Energy Journal, 2018, vol. 33, no. 3, pp. 6-34. doi: 10.1080/21563306.2018.12016723.

7. Kannan S.M., Renuga P., Kalyani S., Muthukumaran E. Optimal capacitor placement and sizing using Fuzzy-DE and Fuzzy-MAPSO methods. Applied Soft Computing, 2011, vol. 11, no. 8, pp. 4997-5005. doi: 10.1016/j.asoc.2011.05.058.

8. Yang X.-S. Firefly Algorithms for Multimodal Optimization. In: Watanabe O., Zeugmann T. (eds) Stochastic Algorithms: Foundations and Applications. SAGA 2009. Lecture Notes in Computer Science, vol. 5792. doi: 10.1007/978-3-642-04944-6_14.

9. Aydin I. A new approach based on firefly algorithm for visionbased railway overhead inspection system. Measurement, 2015, vol. 74, pp. 43-55. doi: 10.1016/j.measurement.2015.07.022.

10. Gao M.-L., Li L.-L., Sun X.-M., Yin L.-J., Li H.-T., Luo D.S. Firefly algorithm (FA) based particle filter method for visual tracking. Optik, 2015, vol. 126, no. 18, pp. 1705-1711. doi: 10.1016/j.ijleo.2015.05.028.

11. Syahputra R., Soesanti I., Ashari M. Performance enhancement of distribution network with DG integration using modified PSO algorithm. Journal of Electrical Systems, 2016, vol. 12 , iss. 1, pp. 1-19.

12. Rezaei M., Ghanbari M. Optimization of sizing and placement of photovoltaic (PV) system in distribution networks considering power variations of $\mathrm{PV}$ and consumers using dynamic particle swarm optimization algorithm (DPSO). Indian Journal of Fundamental and Applied Life Sciences, 2015, vol. 5(S1), pp. 3321-3327.

13. Siavash M., Pfeifer C., Rahiminejad A., Vahidi B. Reconfiguration of smart distribution network in the presence of renewable DG's using GWO algorithm. IOP Conference Series: Earth and Environmental Science, 2017, vol. 83, p. 012003. doi: 10.1088/1755-1315/83/1/012003.

14. Ajenikoko G.A., Ajenikoko O.E.O., Adedoyin A., Badmus B.E., Lawal E.A. Application of genetic algorithm to network feeder reconfiguration in radial distribution system. International journal of advanced scientific and technical research, 2018, vol. 5, no. 8, pp. 15-29. doi: 10.26808/rs.st.i8v5.02.

15. El-Fergany A.A., Abdelaziz A.Y. Cuckoo search-based algorithm for optimal shunt capacitors allocations in distribution networks. Electric Power Components and Systems, 2013, vol. 41, no. 16, pp. 1567-1581. doi: 10.1080/15325008.2013.834525.

16. Mohamed Shuaib Y., Surya Kalavathi M., Christober Asir Rajan C. Optimal capacitor placement in radial distribution system using Gravitational Search Algorithm. International Journal of Electrical Power \& Energy Systems, 2015, vol. 64, pp. 384-397. doi: 10.1016/j.ijepes.2014.07.041.
17. Sambaiah K.S., Jayabarathi T. Optimal allocation of renewable distributed generation and capacitor banks in distribution systems using salp swarm algorithm. International journal of renewable energy research, 2019, vol. 9, no. 1, pp. 96-107.

18. Mohamed E.A., Mohamed A.-A.A., Mitani Y. Hybrid GMSA for optimal placement and sizing of distributed generation and shunt capacitors. Journal of Engineering Science and Technology Review, 2018, vol. 11, no. 1, pp. 55-65. doi: 10.25103/jestr.111.07.

19. Duong M., Pham T., Nguyen T., Doan A., Tran H. Determination of optimal location and sizing of solar photovoltaic distribution generation units in radial distribution systems. Energies, 2019, vol. 12, no. 1, p. 174. doi: 10.3390/en12010174.

20. Sajjadi S.M., Haghifam M.-R., Salehi J. Simultaneous placement of distributed generation and capacitors in distribution networks considering voltage stability index. International Journal of Electrical Power \& Energy Systems, 2013, vol. 46, pp. 366-375. doi: 10.1016/j.ijepes.2012.10.027.

21. Xin J., Zhong J., Yang F., Cui Y., Sheng J. An improved genetic algorithm for path-planning of unmanned surface vehicle. Sensors, 2019, vol. 19, no. 11, p. 2640. doi: 10.3390/s19112640.

22. Khanra A., Pal T., Maiti M.K., Maiti M. Multi-objective four dimensional imprecise TSP solved with a hybrid multiobjective ant colony optimization-genetic algorithm with diversity. Journal of Intelligent \& Fuzzy Systems, 2019, vol. 36, no. 1, pp. 47-65. doi: 10.3233/jifs-172127.

23. Mirjalili S., Gandomi A.H., Mirjalili S.Z., Saremi S., Faris H., Mirjalili S.M. Salp Swarm Algorithm: A bio-inspired optimizer for engineering design problems. Advances in Engineering Software, 2017, vol. 114, pp. 163-191. doi: 10.1016/j.advengsoft.2017.07.002.

24. Mafarja M., Eleyan D., Abdullah S., Mirjalili S. S-Shaped vs. V-Shaped Transfer Functions for Ant Lion Optimization Algorithm in Feature Selection Problem. Proceedings of the International Conference on Future Networks and Distributed Systems - ICFNDS'17, 2017. doi: 10.1145/3102304.3102325.

25. Hegazy A.E., Makhlouf M.A., El-Tawel G.S. Improved salp swarm algorithm for feature selection. Journal of King Saud University - Computer and Information Sciences, 2020, vol. 32, no. 3, pp. 335-344. doi: 10.1016/j.jksuci.2018.06.003.

26. Amrane Y. Optimal reactive power compensation in the transport and distribution networks. Doctoral Thesis, University of Science and Technology Houari Boumediene, Algeria, 2014. 27. Chandramohan S., Atturulu N., Devi R.P.K., Venkatesh B. Operating cost minimization of a radial distribution system in a deregulated electricity market through reconfiguration using NSGA method. International Journal of Electrical Power \& Energy Systems, 2010, vol. 32, no. 2, pp. 126-132. doi: 10.1016/j.ijepes.2009.06.023.

Received 24.06.2020

Chabane Djabali ${ }^{1}$, Ph.D Student,

Tarek Bouktir ${ }^{1}$, Professor,

${ }^{1}$ Department of Electrical Engineering,

University of Ferhat Abbes Setif 1,

19000, Setif, Algeria.

e-mail: Djabali.chabane@gmail.com, tbouktir@univ-setif.dz

How to cite this article:

Djabali C., Bouktir T. Simultaneous allocation of multiple distributed generation and capacitors in radial network using genetic-salp swarm algorithm. Electrical engineering \& electromechanics, 2020, no. 4, pp. 59-66. doi: 10.20998/2074272X.2020.4.08. 